\title{
A MINIATURIZED MEDICAL DEVICE FOR HEALTH MONITORING AND TELEASSISTANCE APPLICATIONS
}

\section{GIORGIO A.}

Politecnico di Bari, Dipartimento di Elettrotecnica ed Elettronica, Via E. Orabona 470125 Bari, ITALY.

${ }^{*}$ Corresponding Author: Email- a.giorgio@poliba.it

Received: December 07, 2011; Accepted: March 15, 2012

\begin{abstract}
Portable Biomedical Devices for health care management are producing a great impact in the monitoring of patients located in areas different from clinical environments such as homes, military bases, ships, and the like. The development of portable telemedicine device is accelerated by new technology like wireless transmission, GPS (Global Positioning System) receiver, Internet application and reduction of electronic device that make it wearable.

The aim of the developed device is the provisioning of extended monitoring for patients under therapy after infarction, data collection in some particular cases, remote consultation and low-cost ECG monitoring for the elderlies unable to announce their failure condition. So the system allows real-time rescue of patients having heart failure and high risk of life .
\end{abstract}

Key words- Medical Device,Health Monitoring, Teleassistance, Global Positioning System.

Citation: Giorgio A. (2012) A Miniaturized Medical Device for Health Monitoring and Teleassistance Applications. International Journal of Bioinformatics Research, ISSN: 0975-3087 \& E-ISSN: 0975-9115, Volume 4, Issue 1, pp.-258-262.

Copyright: Copyright@2012 Giorgio A. This is an open-access article distributed under the terms of the Creative Commons Attribution License, which permits unrestricted use, distribution, and reproduction in any medium, provided the original author and source are credited.

\section{Introduction}

Telemedicine is a term generally used to describe a type of patient care which involves monitoring of a patient's condition by a healthcare worker located at a healthcare facility which is remote with respect to the location of the patient [1]. Demand for electronic patient monitoring systems will appreciably grow in next years, bolstered by technological advances. Wireless multi-parameter monitors and stations will place gains in equipment sales.

Although telemedicine systems have been implemented for many years, Ethernet has just begun to be implemented in the last decade. A much more cost-effective solution would be to take advantage of the already existing Internet. Moreover, the spread of wireless technology allows the development of more telemedicine devices with a low or no cost connections, at short or long distance, and also wearable and easily portable.

From this point of view, telemedicine is defined as the delivery of health care and sharing of medical knowledge over distance using telecommunication means. Thus, telemedicine aims to provide expert-based health care to understaffed remote sites and ad- vanced emergency care through modern telecommunication and information technologies. Nowadays, telemedicine integrates network and medical technology, generally comprising remote diagnosis, expert consultation, information service, online checkups, remote communication, etc. Based on computers and network communication, it implements remote transfer, storage, query, comparison, display, and sharing of video and audio information and medical data of a patient.

The availability of prompt and expert medical care can meaningfully improve health care services at understaffed rural or remote areas. Then, telemedicine, if adequately employed, is capable of providing enormous benefits to society [2]-[6].

One such benefit is that patients can be examined without having to travel to a healthcare facility. This feature is particularly important for patients who live in remote areas who may not be able to easily travel to the nearest healthcare facility, or who need to be examined by a healthcare worker located far away from the patient, in another state, for example.

Another benefit of telemedicine is that it is capable of allowing a 
patient to be examined more often than would be possible if the patient were required to travel to a healthcare facility due to the ease with which it can be administered.

Moreover, telemedicine means also home care of the health; and home care allows a significance improvement of the patient quality of life and a significance reduction of the sanitary expenses [7]. Finally, at a more deep glance, telemedicine deals also with the tele-assistance, i.e. the real time rescue of chronically ill patients and with a high risk of life.

Then, in this paper it is described an innovative portable device useful for tele-assistance and health telemonitoring applications by using the nowdays best known information and communication technologies [8]-[11].

\section{Device Behavioural Specifications and Block Diagram}

The device purpose is the provision of extended monitoring for chronically ill patients, i.e. the patients under therapy after infarction, data collection in some particular cases, remote consultation and low-cost ECG monitoring for the elderlies that are unable to announce their failure condition. So the device allows real-time rescue of patients having heart failure and high risk of life.

The device is based on the SMTP (Simple Mail Transport Protocol) protocol and SMS (Short Message Service); it has been developed with low-cost hardware equipment and it transmits the ECG (ElectroCardioGram) signal and alarm status of a patient. The design has been developed into different hardware and software configurations.

The former configuration, named Pain Button (PB), features GSM/ GPRS wireless technology for data transmission. It is suitable for outdoor use. The block diagram is shown in Fig. (1).

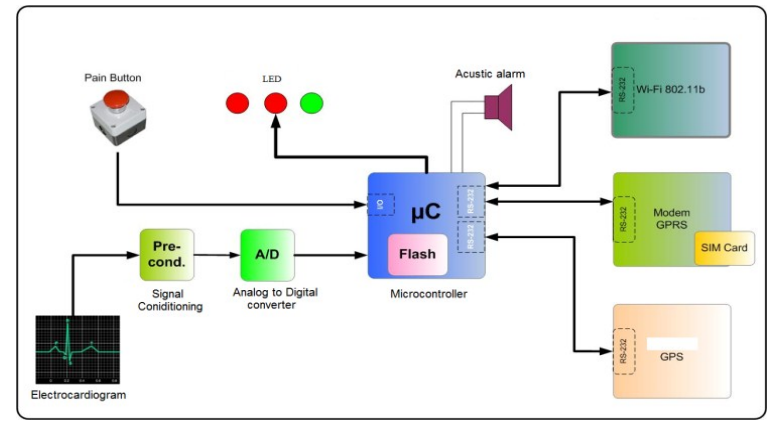

Fig. 1- Pain Button (PB) block diagram

The latter configuration, named Wireless Pain Button (WiPB), features Wi-Fi 802.11g wireless technology (over and above GSM/ GPRS) for data transmission. It is suitable for indoor and outdoor use. In Fig. (2) the block diagram is shown.

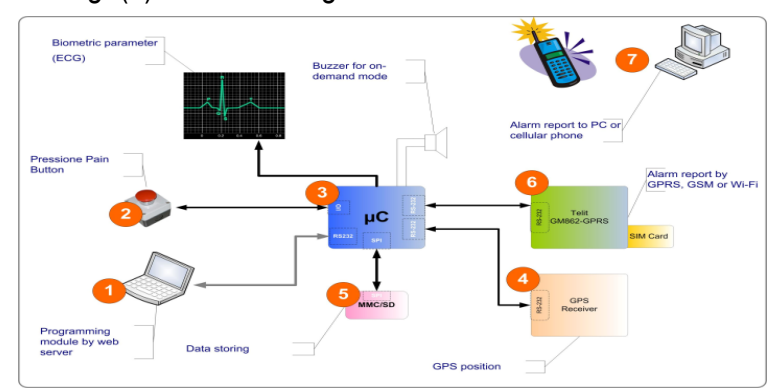

Fig. 2- Wireless Pain Button (WiPB) block diagram
Both configurations are equipped with a GPS receiver to signalling the exact patient position. It is a basic requirement for any telemedicine system, since the GPS is necessary to allow an immediate assistance in case of disease.

Both configurations of the device (PB and WiPB) are equipped with an analog-to-digital front-end to convert health status data and transmit them to the central unit. An external sensor port is provided in patient home communication interface device. The device is able to manage a plurality of external sensors such as those of ECG, blood pressure monitor, blood glucose level monitor, pulse variability monitor, pulse oximeter, and so on. Anyway, to match the essential aim of to be the device portable and wearable, the only ECG device is integrated in PB and WiPB, while all other sensors are external and optional. It is because ECG parameter is the main factor to determine the patient condition and possible risk of life. The system is able to automatically determine heart disease, like tachicardia, bradycardia, arrhythmia and so on, as described follow.

\section{ECG and Heart Disease Detection}

Electrocardiography (ECG) is a transthoracic interpretation of the electrical activity of the heart over time captured and externally recorded by skin electrode [12]. It is a noninvasive recording produced by an electrocardiographic device.

A typical ECG tracing of a normal heartbeat (or cardiac cycle) consists of a $P$ wave, a QRS complex and a T wave. A small $U$ wave is normally visible in 50 to $75 \%$ of ECGs. The baseline voltage of the electrocardiogram is known as the isoelectric line. Typically the isoelectric line is measured as the portion of the tracing following the $T$ wave and preceding the next $P$ wave. Fig. (3) shows a typical ECG waveform.

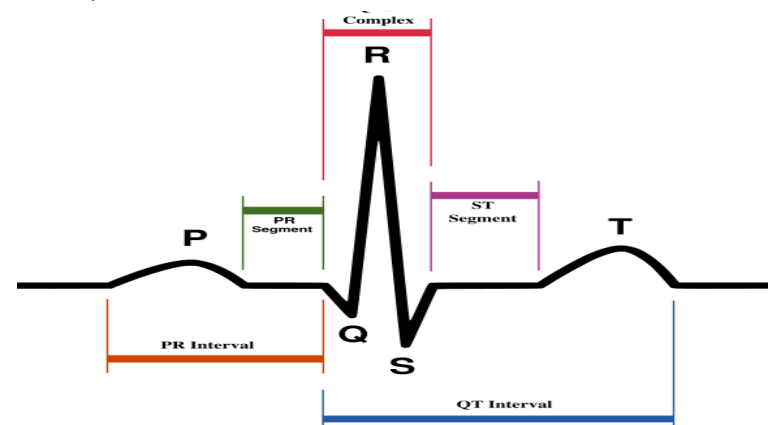

Fig. 3- Electrocardiogram waveform

The shape, length and amplitude of ECG allows to determine the heart condition and possible heart disease. To reveal all ECG parameters, the system uses a QRS detection algorithms based on threshold method [13]-[16]. Since the QRS is the wave complex with higher amplitude, it is a simpler method to calculate the heart beat. The problem associated with this method is right threshold choice. According to [3], the equation (1) has been used:

$$
U_{T H}=\frac{A}{2}+\frac{N}{A} \cdot \ln \left(\frac{P_{0}}{P_{1}}\right)
$$

where $P_{1}$ and $P_{0}$ are respectively the probability to have and not to have a QRS complex, $A$ is the ECG signal amplitude and $N$ is noise variance. So, assuming typical value for this parameter (according to AAMI - Association for the Advancement of Medical 
Instrumentation [17]), it results a threshold of $70 \%$.

This value was the starting point for microprocessor data processing to calculate the heart beat (HB).

When $\mathrm{HB}$ is found it is simple to identify various heart disease like tachycardia $\left(\mathrm{HB}>\mathrm{HB}_{\text {tach }}[\mathrm{bpm}]\right)$ or bradycardia $\left(\mathrm{HB}<\mathrm{HB}_{\text {brady }}\right.$ [bpm]). The parameter $\mathrm{HB}_{\text {tach }}$ and $\mathrm{HB}_{\text {brady }}$ could change from patient to patient [18]. So the device implements a simply web interface to change this parameter.

To identify arrhythmia, beyond tachicardia and bradycardia, we defined an arrhythmia factor as follow:

$$
\alpha=\frac{\Delta T_{1}}{\Delta T_{2}}
$$

where $\Delta T 1$ and $\Delta T 2$ are respectively the time between three consecutive QRS complex, like Fig. (4) shows.

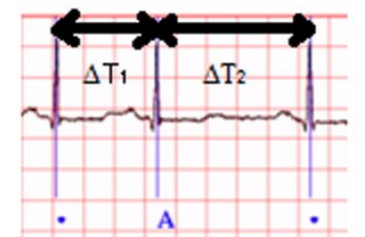

Fig. 4- arrhythmia factor and detection method

In an ideal case, we will have an arrhythmic complex when $a \neq 1$, while in the real case, we define the following range:

$$
\Delta \mathrm{T} 1<(\Delta \mathrm{T} 1+\Delta \mathrm{T} 2) \cdot 40 \%
$$

that is,

$$
(a<0.25) \text { and }(a>4)
$$

Operation Example: Wireless Alarm And Data Transmission The device can send automatic and manual alarm status reports. In case of illness the patient presses Pain Button to signalling his condition (manual status report). Otherwise, PB/WiPB acquires and processes ECG signal and reports automatically abnormal cardiac behaviour (automatic status report).

The device performs analog-to-digital conversion and analyzes any variation in shape, duration, amplitude and frequency of ECG in real-time.

Digital data are stored into internal flash memory or in an external memory card MMC/SD, based on overall data.

In order to ensure the resilience of the system, PB/WiPB uses three different transmission technology [9]-[10], [19]: GSM to send SMS (Short Message Service), GPRS and Wi-Fi $802.11 \mathrm{~g}$ to send verbose alarm report (ECG signal and GPS coordinate) to PC. Fig. (5) shows all possible transmission method implemented in $\mathrm{PB} / \mathrm{WiPB}$.

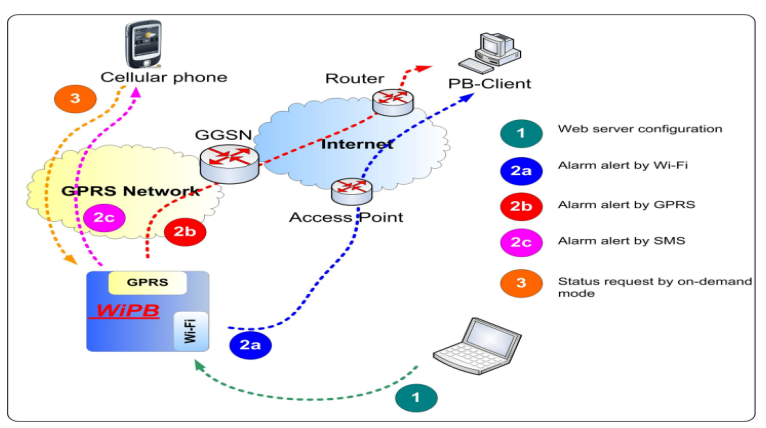

Fig. 5- Data transmission from $\mathrm{PB}$ to $\mathrm{PC}$ and cellular phone
Thanks to Wi-Fi 802.11g interface, it is possible to configure the device without using any cable and while the patient wears it, even directly by clinic. Moreover, the system integrates a web server to make simpler the configuration step. Simply connecting trough a web browser (like Internet Explore, Firefox or Safari) and inserting the IP address of the device, it is possible to enter the

\begin{tabular}{|c|c|}
\hline \multicolumn{2}{|c|}{ Wireless Pain Button } \\
\hline \multicolumn{2}{|r|}{ SMS CONFIGURATION } \\
\hline \multicolumn{2}{|l|}{ SMS Enable } \\
\hline$\odot$ Enable & $O_{\text {Disable }}$ \\
\hline Recipient Cellular Phone & 3401234567 \\
\hline \multicolumn{2}{|r|}{ EMAIL CONFIGURATION } \\
\hline \multicolumn{2}{|l|}{ EMAIL Enable } \\
\hline - Enable & $C$ Disable \\
\hline Recipient Email & email@poliba.it \\
\hline \multicolumn{2}{|l|}{ Submit } \\
\hline Server SMTP & $\Gamma$ \\
\hline SMTP Port & \\
\hline Sender Email & \\
\hline Username & \\
\hline Password & 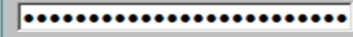 \\
\hline Email Authentication & $\Gamma$ checked / \\
\hline Debug & \\
\hline Submit & \\
\hline
\end{tabular}
ECG parameter, phone number to send the alarm report or email address of PB-Client, like shown in Fig. (6).

Fig. 6- Web Interface to programming device by Wi-Fi

To allow the security access to the system, all web pages access has been protected with username and password.

In the described device data transmission by GPRS or Wi-Fi $802.11 \mathrm{~g}$ uses application layer protocol (SMTP) based on reliable TCP protocol as it offers permanent connection channels and data integrity.

Having described the behavioural specifications, the block diagram and the principle of operation of the device, in the following sections design details will be given, referring to figures 1 and 2 .

\section{Central Processing Unit}

The main component of the device is the microcontroller $(\mu \mathrm{C})$ that supervises all other parts. The choice of $\mu \mathrm{C}$ is fundamental for all design.

Nowadays, many design solutions exist. Systems based on embedded processors such as monolithic, Digital Signal Processors (DSPs), ARM processors, and FPGA (Field Programmable Gate Array) are good solutions.

To perform the best choice, it's important to take into account many matters, among which the available program memory, performance in terms of MIPS (Million Instruction Per Seconds), availability of analog-to-digital (ADC) converter and its resolution, digital peripheral like UART (Universal Asynchronous Receiver 
Transmitter), USB (Universal Serial Bus) or Ethernet. The MIPS performance is a basic features since the microcontroller has to process the data in real-time and transmit it to the clinic.

Among all possible solution, we focus on two different brand: Microchip [20] and Tibbo [21] Technologies. The former is suitable to develop the PB configuration, while the latter to develop the WiPB configuration.

Microchip has a wide range of product named PIC and dsPIC. We choice the model PIC18F4550 as the base model, but thanks to the code compatibility is possible to upgrade the system without change the written firmware; in our analysis we evaluate, also, the possibility of using the PIC18F97J60 family that has embedded Ethernet. The main advantages of using PIC microcontroller are the low power consumption, high integration and low cost.

Tibbo solution is EM1000 microprocessor. The main advantage is the possibility to integrate Wi-Fi $802.11 \mathrm{~g}$ transmitter. So it is a good solution for WiPB.

\section{Management Software}

To be useful, the device has to be supervised by an integrated client software. The software for PB (named PB-Client) was developed using Delphi language. It is capable to supervising more than one PB/WiPB, showing for each PB/WiPB alarm status and type, ECG trace (see Fig. (7)) and geographical position.

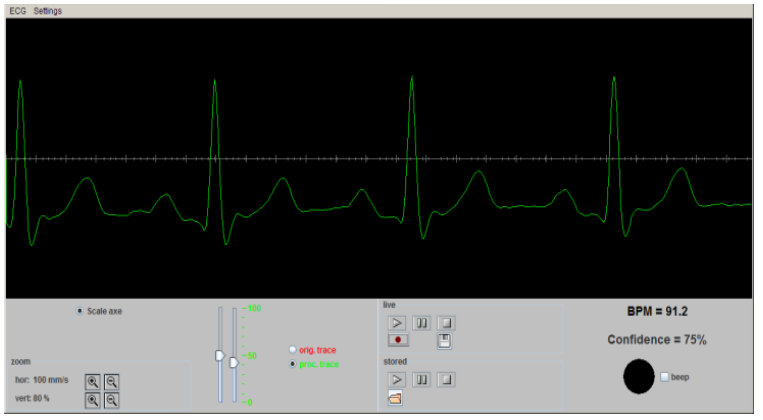

Fig. 7- ECG trace shown by PB-Client software.

\section{Global Positioning System}

The device has to automatically notify an illness. Beyond this main requirement, it is necessary, for example in case of heart failure or high risk of life, to know exactly where the patient is. In this way it's possible to reduce the rescue time. So the proposed device embodies a GPS (Global Positioning System) receiver to acquire the real-time patient position [19]. It has been also developed a client-side software with a Graphical User Interface (GUI), PBClient, able to capture alarm status report, showing exact patient position by interaction with software for geographical visualization and analysis, like Microsoft Mappoint and Google Maps (however it is possible to integrate with other software for geographical visualization). So the remote observer can monitor the patient ECG and his position, simply by typing the connection parameter.

\section{GSM/GPRS Transmission and GPS}

Another main requirements in developing of the device is the compactness. So has been chosen a GSM/GPRS transmission system: Telit GM862 [22]. It combines superior performance in quad-band GSM/GPRS modem functionality with the latest 20channel high sensitivity SiRFstarl|I ${ }^{\mathrm{TM}}$ single-chip GPS receiver.
So only one system feature not only GSM/GPRS functionality but also GPS receiver. The GPS receiver features low power consumption with position resolution accuracy of less than $2.5 \mathrm{~m}$, SBAS (WAAS and EGNOS). It allow high sensitivity for indoor fixes, so we can get the patient position not only when it is outdoor but also indoor.

GM862 integrated TCP/IP protocol stack, allowing us to develop web application like sending email as alarm status report.

\section{Prototype of the Device}

A working prototype of PB/WiPB has been realized, as shown in Fig. (8). The dimensions are $9.5 \times 5.5 \mathrm{~cm}^{2}$, so it is easily wearable, such as a common cellular phone.

PB and WiPB consists of a set of WiFi/GPRS-enabled instruments that communicate wirelessly over Internet. Equipped with a miniature circuit board, devices such as a ECG monitor or other external sensors can communicate with clinic until they are within range of an access point (only in WiPB configuration); if no access points are found, the device uses a GPRS connection to access to Internet; even if no GPRS signal is detected, the device uses the GSM network. It sends the encrypted data to the clinic, which consists of multiple redundant servers and makes the data available to authorized specialist via web server. The same advanced communication technologies allow the transmission of data dealing with implantable devices such as defibrillators and pacemaker.

The PB/WiPB is easy to operate, so patients with limited abilities or patient with heart disease can use it without difficulty: they only have to press the pain button if needed, but even if they can't press this button, the device automatically sends data.

Due to its features the PB should be a very nice solution in the future to improve the quality of life and the life expectancy of diseased, high risk subjects.

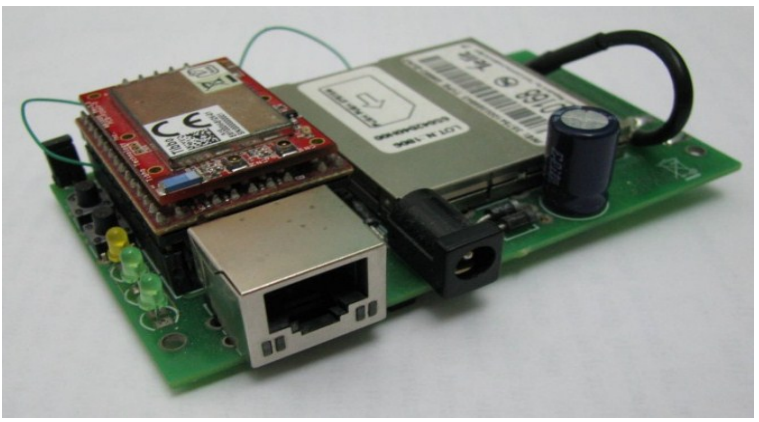

Fig. 8- WiPB prototype (dimension: $9.5 \mathrm{~cm} \times 5.5 \mathrm{~cm}$ )

\section{Conclusions}

In this paper an innovative device for telemonitoring of the health and teleassistance applications has been described.

The device is an application of all the nowdays best known ICT and appears to be very promising in terms of usefulness, compactness (wearability) and capabilities for real time rescue of patients having a high risk of life.

\section{References}

[1] http://it.wikipedia.org/wiki/Telemedicina.

[2] Weston C.F.M., Penny W.J., Julian D.J. (1994) British Medical 
Journal, 308, 767-771.

[3] Evans T. (1998) British Medical Journal, 316, 1031-1032.

[4] Sandler D.A (1999) British Medical Journal, 318, 1553.

[5] Canto J.G., Rogers W.J., Bowlby L.J., French W.J., Pearce D. J., Weaver W.D. (1997) Journal of the American College of Cardiology, 29, 498-505.

[6] Stults K.R., Brown D.D., Schug V.L., Bean J.A. (1984) The New England Journal of Medicine, 310(4), 219-223.

[7] Barro S., Presedo J., Castro D., Fernandez-Delgado M., Fraga S., Lama M., Vila J. (1999) IEEE Engineering in Medicine and Biology, 18(4), 80-88.

[8] Clapp G., Brown S.J. (2008) Patent: US20080058615.

[9] Ng H.S., Sim M.L., Tan C.M. and Wong C.C. (2006) BT Technologies Journal, 4(2), 130.

[10]Surana S., Patra R., Nedevschi S., Brewer E. (2008) IEEE Computer Society, 41(6), 48-56.

[11] Giorgio A. Telemedicine: techniques and applications, In Tech ed., ISBN 979-953-307-008-6

[12] http://it.wikipedia.org/wiki/Elettrocardiogramma.

[13]Pan J., Tompkins W.J. (1985) IEEE Transaction on biomedical engineering, 32(3), 230-236.

[14]Ruha A., Sallinen S.S., Nissilä S. (1997) IEEE Transaction on biomedical engineering, 44(3), 159-167.

[15]Nygards M., Sornmo L. (1981) Conference Computers in cardiology, published by IEEE Computers Society, 347-350.

[16]Ligtenberg A., Kunt M. (1983) Computer cardiology, 16, 273286.

[17]MIT-BIH Database Distribution, Massachusetts Institute of Technology, 77 Massachusetts Avenue, Room 20A-113, Cambridge, MA 02139.

[18]"Standard for cardiac monitors, heart rate meters and alarms (draft) (1981) Association for the advancement of medical instrumentation (AAMI).

[19]http://www.gps-practice-and-fun.com/telemedicine.html.

[20]http://www.microchip.com/wwwproducts/Devices.aspx? dDocName $=$ en010296.

[21] http://www.tibbo.com/em1000.php.

[22]http://www.telit.com/. 